\title{
CONSIDERAÇÕES ANESTÉSICAS EM PACIENTES PEDIÁTRICOS COM TRANSTORNO DO ESPECTRO AUTISTA
}

\author{
ANESTHESIC CONSIDERATIONS IN PEDIATRIC PATIENTS WITH AUTISM SPECTRUM \\ DISORDER
}

Amanda Gosson de Melo Dibe $\mathrm{MD}^{1}$; Cynthia Cristina Bastia Vieira de Souza MD, MSc²; Raphael Klênio Confessor de Sousa $\mathrm{MD}^{3}$; Wallace Andrino Silva MD, PhD ${ }^{4}$

1. Médica anestesiologista formada pelo Hospital Universitário Onofre Lopes/UFRN.

2. Médica anestesiologista do Hospital Univesitário Onofre Lopes/UFRN. Mestrado pelo Centro de Ciências da Saúde/UFRN.

3. Médico anestesiologista do Hospital Univesitário Onofre Lopes/UFRN. Título Superior em Anestesiologia pela Sociedade Brasileira de Anestesiologia (SBA). Instrutor do Centro de Ensino e Treinamento (CET) SBA do HUOL.

4. Médico anestesiologista do Hospital Universitário Onofre Lopes/UFRN. Doutorado pela FMUSP, Título Superior de Anestesiologia pela SBA. Responsável pelo CET SBA do HUOL.

Artigo produzido na Divisão de Anestesiologia do Hospital Universitário Onofre Lopes (UFRN).

Financial support: None.

Conflicts of interest: None.

Address for correspondence: Hospital Universitário Onofre Lopes, Divisão de Anestesiologia. Av. Nilo Peçanha, 620, $3^{\circ}$ subsolo, Petrópolis, Natal/RN CEP:59012-300.

Submitted: feb 14; accepted after revision, sep 16, 2021.

\section{ABSTRACT}

Introduction: Patients with autism spectrum disorder, in most cases, especially children, have exacerbated sensitivity, and this fact should be considered when anesthesia is required. Aim: To conduct a literature review trying to compile the main studies related to the anesthetic implications in pediatric patients with autism spectrum disorder. Method: The basis used for the selection of works was PUBMED. The search strategy established was as follows: ((anesthesia [MeSH Terms]) AND (autism spectrum disorder [MeSH Terms])) AND (child [MeSH Terms]). Results: The search initially identified 34 articles, of which 12 articles were considered for review. Conclusion: Autism spectrum disorder generates several practical difficulties for the application of anesthesia. It creates the need for sedation in a wide range of medical procedures. Physical restraint and forced application of anxiolytics to facilitate anesthetic applications, are no longer ethically well regarded, and should only be used as a last resource. Many of the papers mention that a greater interaction with parents or caregivers on the mental particularity of each patient generates good results. Moreover, the training of health professionals on autism and its behavioral consequences is extremely important for anesthesia, surgical procedures and exams achieve the desired success.

Key words: Anesthesia; Child; Autism Spectrum Disorder; Autism. 


\section{RESUMO}

Introdução: Pacientes autistas, na maioria dos casos, especialmente as crianças, apresentam sensibilidade exacerbada, o que deve ser considerado quando existe a necessidade de uma anestesia. Objetivo: Realizar uma revisão da literatura buscando compilar os principais estudos relacionados às implicações anestésicas em pacientes pediátricos com transtorno do espectro autista (TEA). Método: A base utilizada para seleção dos trabalhos foi a PUBMED. A estratégia de busca estabelecida foi a seguinte: ((anesthesia[MeSH Terms]) AND (autism spectrum disorder[MeSH Terms])) AND (child[MeSH Terms]). Resultados: A busca identificou inicialmente 34 artigos, sendo considerados 12 artigos para a revisão. Conclusão: $O$ transtorno do espectro autista gera diversas dificuldades práticas para a aplicação de anestesias. Criando, inclusive, a necessidade de sedação em uma ampla gama de procedimentos médicos. Contenção física e uso forçado de ansiolíticos para facilitar aplicações anestésicas, já não são mais eticamente bem vistos, devendo ser utilizados apenas em último caso. Muitos dos trabalhos citam que uma interação maior com os pais ou cuidadores sobre as especificidades mentais de cada paciente geram bons resultados. Além disso, 0 treinamento de profissionais da saúde sobre o autismo e seus desdobramentos comportamentais são de extrema importância para que as anestesias, os procedimentos cirúrgicos e exames obtenham o sucesso desejado.

Palavras-chave: Anestesia; Criança; Transtorno do Espectro Autista; Autismo.

\section{INTRODUÇÃO}

O transtorno do espectro autista (TEA) descreve indivíduos com uma combinação específica de deficiência na comunicação social, comportamentos repetitivos, interesses altamente restritos e / ou comportamentos sensoriais que se manifestam precocemente. A prevalência mundial de autismo é de pouco menos de $1 \%$, mas as estimativas são mais altas em países de alta renda. Embora a presença de doença cerebral macroscópica não seja característica do autismo, diferenças anatômicas e funcionais sutis foram observadas em estudos post-mortem, de neuroimagem e eletrofisiológicos. Inicialmente, esperava-se que a medição precisa dos fenótipos comportamentais levasse a subtipos genéticos específicos, mas os achados genéticos se aplicaram principalmente a grupos heterogêneos que não são específicos ao autismo. As intervenções psicossociais em crianças podem melhorar comportamentos, como atenção conjunta, linguagem e engajamento social, que podem afetar o desenvolvimento futuro e reduzir a gravidade dos sintomas ${ }^{1}$.

Pacientes autistas, na maioria dos casos, especialmente as crianças, apresentam sensibilidade exacerbada, o que deve ser considerado quando existe a necessidade de uma anestesia. Os medicamentos antipsicóticos, por exemplo a risperidona, podem causar hipotensão quando utilizados em conjunto com anestesia geral, podendo apresentar propriedades pró-arrítmicas. A utilização prévia de psicoestimulantes pode aumentar a necessidade de dose de sedativo durante a anestesia, aumentar o risco de 
hipertensão e arritmias, além de diminuir o limiar convulsivo e interagir com vasopressores. Existe o risco de hipertensão significativa e súbita quando metilfenidato e agentes halogenados são usados concomitantemente ${ }^{2}$.

Frequentemente, a primeira interação do anestesista com o paciente é no dia do procedimento. Essa abordagem não fornece uma preparação adequada e o estabelecimento de uma relação ideal entre paciente-pais-provedor. Nesse sentido, revisar a literatura buscando elucidar as principais implicações relacionadas às anestesias em crianças portadoras do transtorno do espectro autista, fornecerá informações que poderão ser utilizadas para sistematizar o atendimento a esse grupo especial de pacientes, reduzindo as complicações e os possíveis contratempos.

O objetivo desse artigo é realizar uma revisão da literatura buscando compilar os principais estudos relacionado às implicações anestésicas em pacientes pediátricos com transtorno do espectro autista, auxiliando na sistematização do atendimento a esse grupo de pacientes.

\section{MÉTODOS}

Este trabalho é caracterizado como uma pesquisa exploratória, visto que avalia estudos já publicados. A base utilizada para seleção dos artigos foi a PUBMED. A estratégia de busca estabelecida foi a seguinte: ((anesthesia[MeSH Terms]) AND (autism spectrum disorder[MeSH Terms])) AND (child[MeSH Terms]), limitando a busca dos artigos dos últimos 10 anos (2010-2020). O principal critério de inclusão foi discutir efetivamente as implicações anestésicas em pacientes pediátricos com transtorno do espectro autista. Foram excluídos artigos que destoavam do tema central, assim como possíveis editoriais. 
Considerações anestésicas em pacientes pediátricos com transtorno do espectro autista

Dibe AGM, et al

\section{RESULTADOS}

A busca identificou inicialmente 34 artigos. Após a leitura dos títulos e resumos, 21 trabalhos foram excluídos por destoarem do tema central estabelecido para a presente revisão e um foi eliminado por se tratar de um editorial. Após esta etapa, os 12 artigos restantes foram lidos em sua íntegra, resumidos, e apresentados na seção a seguir em ordem cronológica considerando a data de publicação. O processo de seleção está representado na Figura 1.
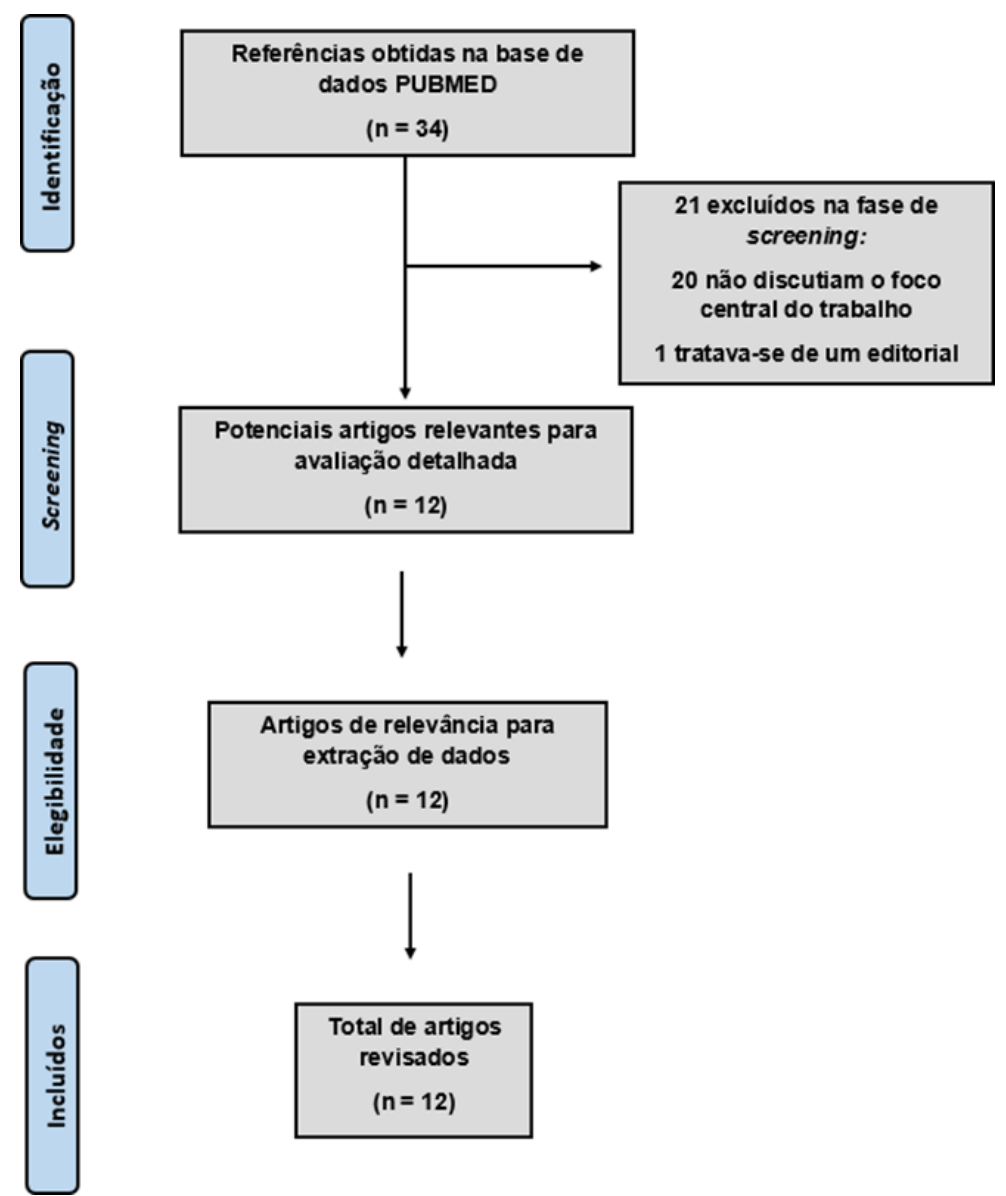

Figura 1 - Processo de seleção dos artigos revisados 
Considerações anestésicas em pacientes pediátricos com transtorno do espectro autista

Dibe $A G M$, et al

A Tabela 1 traz o resumo das principais informações relacionadas aos 12 artigos selecionados para revisão.

Tabela 1 - Resumo das principais informações relacionadas aos artigos

\begin{tabular}{|c|c|c|c|c|c|}
\hline Título & Autor & Ano & Desenho & Terapêutica & Desfecho \\
\hline $\begin{array}{l}\text { Special care dentistry: } \\
\text { Midazolam conscious sedation } \\
\text { for patients with neurological } \\
\text { diseases. }\end{array}$ & Capp et al. & 2010 & $\begin{array}{l}\text { Estudo } \\
\text { Descritivo }\end{array}$ & $\begin{array}{l}\text { Midazolam } \\
\text { (intravenoso ou } \\
\text { intramuscular) e } \\
\text { lidocaína }\end{array}$ & $\begin{array}{l}\text { O midazolam demonstrouser eficaz em } 89 \% \text { da amostra para procedimentos } \\
\text { odontológicos em pacientes com distúrbios neurológicos e comportamentais, mas foi } \\
\text { menos eficaz em pacientes com autismo }(p<0.05)\end{array}$ \\
\hline $\begin{array}{l}\text { Anaesthesia and the autistic } \\
\text { child. }\end{array}$ & Bagshaw & 2011 & $\begin{array}{l}\text { Estudo } \\
\text { Descritivo }\end{array}$ & $\begin{array}{l}\text { Plano de cuidados para } \\
\text { ser aplicado em pacientes } \\
\text { autistas a serem } \\
\text { submetidos a } \\
\text { procedimentos } \\
\text { anestésicos. }\end{array}$ & $\begin{array}{l}\text { O plano incluiu a necessidade de contato prévio com paciente, acolhimento do } \\
\text { mesmo em local calmo e por pouco tempo, discutir com os cuidadores e outros } \\
\text { provedores necessidade de medicação pré-anestesica, etc }\end{array}$ \\
\hline $\begin{array}{l}\text { Autism spectrum disorder } \\
\text { (ASD) and its perioperative } \\
\text { management. }\end{array}$ & $\begin{array}{l}\text { Taghizade } \\
\text { h et al. }\end{array}$ & 2015 & $\begin{array}{l}\text { Artigo de } \\
\text { Revisão }\end{array}$ & $\begin{array}{l}\text { Manejo perioperatório de } \\
\text { pacientes com transtorno } \\
\text { do espectro autista }\end{array}$ & $\begin{array}{l}\text { Algumas estratégias simples que melhorariam o cuidado e diminuiriam a ansiedade } \\
\text { dos pacientes autistas, como histórias sociais, uso de tablets, jogos para distração, } \\
\text { necessidade de fazer o pós-operatório imediato em local confortável etc. }\end{array}$ \\
\hline $\begin{array}{l}\text { Retrospective Analysis of } \\
\text { Decreasing the Use of } \\
\text { Anesthesia in Pediatric } \\
\text { Audiology: A Preliminary Study. }\end{array}$ & $\begin{array}{l}\text { Padish- } \\
\text { Clarin \& } \\
\text { Hawkins }\end{array}$ & 2015 & $\begin{array}{l}\text { Estudo } \\
\text { Descritivo }\end{array}$ & - & $\begin{array}{l}\text { Os processos que foram observados neste estudo resultaram na eliminação da } \\
\text { necessidade do uso de anestesia para avaliação da audição em grande parte dos } \\
\text { pacientes encaminhados. }\end{array}$ \\
\hline $\begin{array}{l}\text { Perioperative considerations in } \\
\text { children with autism spectrum } \\
\text { disorder. }\end{array}$ & $\begin{array}{l}\text { Vlassakov } \\
\quad \text { a \& } \\
\text { Emmanoui } \\
\text { I }\end{array}$ & 2016 & $\begin{array}{l}\text { Artigo de } \\
\text { Revisão }\end{array}$ & $\begin{array}{l}\text { Manejo perioperatório de } \\
\text { pacientes com TEA }\end{array}$ & $\begin{array}{l}\text { A familiaridade com as especificidades comportamentais de cada paciente com TEA e } \\
\text { esforços para aliviar seu estresse seriam importantes para um curso perioperatório } \\
\text { tranquilo e poucos eventos adversos. }\end{array}$ \\
\hline $\begin{array}{l}\text { Management of Children with } \\
\text { Autism Spectrum Disorder in } \\
\text { the Anesthesia and } \\
\text { Radiographic Context. }\end{array}$ & $\begin{array}{l}\text { Berglund. } \\
\text { et al. }\end{array}$ & 2017 & $\begin{array}{c}\text { Estudo } \\
\text { Prospectivo }\end{array}$ & $\begin{array}{l}\text { Elaboração de diretrizes } \\
\text { através do método Delphi } \\
\text { visando melhorar os } \\
\text { cuidados de crianças com } \\
\text { TEA submetidas a } \\
\text { procedimentos } \\
\text { radiológicos }\end{array}$ & $\begin{array}{l}\text { As aplicações de anestesia e radiologia que envolvem crianças com TEA requerem um } \\
\text { planejamento prévio, atendendo às necessidades individuais de cada criança }\end{array}$ \\
\hline
\end{tabular}

Continua... 
Considerações anestésicas em pacientes pediátricos com transtorno do espectro autista

Dibe AGM, et al

\section{Continuação}

\begin{tabular}{|c|c|c|c|c|c|}
\hline Título & Autor & Ano & Desenho & Terapêutica & Desfecho \\
\hline $\begin{array}{l}\text { Benefits of an individualized } \\
\text { perioperative plan for children } \\
\text { with autism spectrum disorder. }\end{array}$ & $\begin{array}{l}\text { Swartz et } \\
\text { al. }\end{array}$ & 2017 & $\begin{array}{l}\text { Estudo } \\
\text { Descritivo }\end{array}$ & $\begin{array}{l}\text { Descrição de plano, } \\
\text { baseado em revisão de } \\
\text { prontuários, para fornecer } \\
\text { sedação pré-operatória } \\
\text { estratificada pelo nível de } \\
\text { gravidade do TEA }\end{array}$ & $\begin{array}{l}\text { O plano foi útil para orientar as escolhas de sedação, visto que a cooperação não } \\
\text { diferiu entre crianças sedadas e não sedadas em qualquer nível de gravidade }\end{array}$ \\
\hline $\begin{array}{l}\text { Procedural sedation in children } \\
\text { with autism spectrum } \\
\text { disorders: A survey of current } \\
\text { practice patterns of the society } \\
\text { for pediatric sedation members }\end{array}$ & $\begin{array}{l}\text { Kamat et } \\
\text { al. }\end{array}$ & 2018 & $\begin{array}{l}\text { Estudo } \\
\text { Descritivo }\end{array}$ & - & $\begin{array}{l}\text { Melhor compreensão dos recursos necessários, a padronização de estratégias de } \\
\text { gestão comportamental e abordagens farmacológicas e o desenvolvimento de } \\
\text { protocolos otimizariam o atendimento de pacientes com TEA }\end{array}$ \\
\hline $\begin{array}{l}\text { A Comparison of Safety and } \\
\text { Efficacy of Dexmedetomidine } \\
\text { and Propofol in Children with } \\
\text { Autism and Autism Spectrum } \\
\text { Disorders Undergoing Magnetic } \\
\text { Resonance Imaging. }\end{array}$ & $\begin{array}{l}\text { Abulebda } \\
\text { et al. }\end{array}$ & 2018 & $\begin{array}{l}\text { Estudo de } \\
\text { Coorte } \\
\text { Retrospectiv } \\
\quad 0\end{array}$ & $\begin{array}{l}\text { Propofol e } \\
\text { dexmedetomidina }\end{array}$ & $\begin{array}{l}\text { Tanto o propofol quanto a dexmedetomidina mostraram-se medicamentos } \\
\text { adequados e seguros na sedação de crianças autistas submetidas à ressonância } \\
\text { magnética. }\end{array}$ \\
\hline $\begin{array}{l}\text { Parental Satisfaction with } \\
\text { Ambulatory Anesthesia during } \\
\text { Dental Treatment for Disabled } \\
\text { Individuals and Their } \\
\text { Preference for Same in Future. }\end{array}$ & $\begin{array}{l}\text { Ohtawa et } \\
\text { al. }\end{array}$ & 2019 & $\begin{array}{l}\text { Estudo } \\
\text { Descritivo }\end{array}$ & & $\begin{array}{l}\text { Nos casos em que indivíduos com deficiência receberam anestesia geral } \\
\text { anteriormente durante o tratamento odontológico, os pais preferiram a anestesia } \\
\text { geral durante os tratamentos futuros. }\end{array}$ \\
\hline $\begin{array}{l}\text { Behavioral training and } \\
\text { mirroring techniques to } \\
\text { prepare elective anesthesia in } \\
\text { severe Autistic spectrum } \\
\text { disorder patients: An } \\
\text { illustrative case and review. }\end{array}$ & $\begin{array}{l}\text { Mellado- } \\
\text { Cairet et } \\
\text { al. }\end{array}$ & 2019 & $\begin{array}{l}\text { Relato de } \\
\text { Caso }\end{array}$ & $\begin{array}{l}\text { Demonstração em } \\
\text { espelho associada ao } \\
\text { reforço positivo na } \\
\text { preparação da criança } \\
\text { autista para anestesia } \\
\text { geral. }\end{array}$ & $\begin{array}{l}\text { A comunicação precoce com os cuidadores seria necessária para estabelecer que } \\
\text { pacientes poderiam se beneficiar desse treinamento comportamental. }\end{array}$ \\
\hline $\begin{array}{l}\text { Enhanced perioperative } \\
\text { management of children with } \\
\text { autism: a pilot study. }\end{array}$ & $\begin{array}{l}\text { Whippey } \\
\text { et al. }\end{array}$ & 2019 & $\begin{array}{l}\text { Estudo } \\
\text { Prospectivo }\end{array}$ & $\begin{array}{l}\text { Plano de cuidados } \\
\text { perioperatórios } \\
\text { multidisciplinares para } \\
\text { crianças com autismo } \\
\text { grave. }\end{array}$ & $\begin{array}{l}\text { O plano proposto seria viável e altamente aceito na instituição onde o estudo } \\
\text { ocorreu. A natureza individual dos planos de ansiólise foi considerada um ponto forte } \\
\text { do protocolo. }\end{array}$ \\
\hline
\end{tabular}

Continua... 
Considerações anestésicas em pacientes pediátricos com transtorno do espectro autista

Dibe AGM, et al

\section{Continuação}

\begin{tabular}{|l|c|c|c|l|l|}
\hline \multicolumn{1}{|c|}{ Título } & Autor & Ano & Desenho & Terapêutica & \multicolumn{1}{|c|}{ Desfecho } \\
\hline $\begin{array}{l}\text { Retrospective Analysis of } \\
\text { Decreasing the Use of } \\
\text { Anesthesia in Pediatric } \\
\text { Audiology: A Preliminary Study. }\end{array}$ & $\begin{array}{l}\text { Padish- } \\
\text { Clarin \& } \\
\text { Hawkins }\end{array}$ & 2015 & $\begin{array}{c}\text { Estudo } \\
\text { Descritivo }\end{array}$ & $\begin{array}{l}\text { Os processos que foram observados neste estudo resultaram na eliminação da } \\
\text { necessidade do uso de anestesia para avaliação da audição em grande parte dos } \\
\text { pacientes encaminhados. }\end{array}$ \\
\hline $\begin{array}{l}\text { Perioperative considerations in } \\
\text { children with autism spectrum } \\
\text { disorder. }\end{array}$ & $\begin{array}{c}\text { Vlassakov } \\
\text { a \& } \\
\text { Emmanoui } \\
\text { I }\end{array}$ & 2016 & $\begin{array}{c}\text { Artigo de } \\
\text { Revisão }\end{array}$ & $\begin{array}{l}\text { Manejo perioperatório de } \\
\text { pacientes com TEA }\end{array}$ & $\begin{array}{l}\text { A familiaridade com as especificidades comportamentais de cada paciente com TEA e } \\
\text { esforços para aliviar seu estresse seriam importantes para um curso perioperatório } \\
\text { tranquilo e poucos eventos adversos. }\end{array}$ \\
\hline $\begin{array}{l}\text { Management of Children with } \\
\text { Autism Spectrum Disorder in } \\
\text { the Anesthesia and } \\
\text { Radiographic Context. }\end{array}$ & $\begin{array}{c}\text { Berglund. } \\
\text { et al. }\end{array}$ & 2017 & $\begin{array}{l}\text { Estudo } \\
\text { Prospectivo }\end{array}$ & $\begin{array}{l}\text { Elaboração de diretrizes } \\
\text { através do método Delphi } \\
\text { visando melhorar os } \\
\text { cuidados de crianças com } \\
\text { TEA submetidas a } \\
\text { procedimentos } \\
\text { radiológicos }\end{array}$ & $\begin{array}{l}\text { As aplicações de anestesia e radiologia que envolvem crianças com TEA requerem um } \\
\text { planejamento prévio, atendendo às necessidades individuais de cada criança }\end{array}$ \\
\hline
\end{tabular}


Considerações anestésicas em pacientes pediátricos com transtorno do espectro autista Dibe AGM, et al

\section{DISCUSSÃO}

Capp e colaboradores avaliaram a eficácia do midazolam em pacientes com doenças neurológicas (encefalopatia, autismo e epilepsia) que foram encaminhados para tratamento odontológico. Quarenta e cinco sedações foram realizadas com midazolam (intramuscular 0,2-0,3 mg / $\mathrm{kg}$ ou intravenoso 0,1 $\mathrm{mg} / \mathrm{kg}$ ) e em todos foi feita anestesia local com lidocaína 2\% (0,5-2 ml). Durante o procedimento odontológico, os comportamentos foram analisados e classificados em 3 categorias: A (indiferente), B (reagiu, mas permitiu o tratamento) e $C$ (não permitiu o tratamento). Como resultados, os autores relataram que a classificação final dos pacientes foi: A 22 (49\%), B 18 (40\%) e $C 5(11 \%)$ e que os pacientes com encefalopatia tiveram os melhores resultados de sedação de acordo com a classificação proposta $(p<0,05)$. A conclusão a que o grupo chegou foi de que o midazolam demonstrou ser eficaz em $89 \%$ desta amostra para procedimentos odontológicos em pacientes com distúrbio neurológicos e comportamentais, mas foi menos eficaz em pacientes com autismo $(p<0,05)^{3}$.

Bagshaw chamou atenção para o fato de haver pouca literatura na prática anestésica pediátrica que seria relevante para crianças com TEA. Para essa população, seria recomendado que os profissionais envolvidos no caso ganhassem a confiança do paciente, com ou sem pré-medicação, pois pacientes com TEA, normalmente não apresentam as mesmas alterações neuropsicossociais e devem ter seus tratamentos individualizados de acordo com suas necessidades. Um estudo realizado na Austrália identificou algumas necessidades e montou um plano para ser seguido com pacientes autistas que seriam submetidos a procedimentos anestésicos. Esse plano inclui a necessidade de contato prévio com paciente, acolhimento do paciente em local calmo e que não necessite esperar por muito tempo pelo procedimento, pois mudanças de rotinas geram ansiedade e agitação; discutir com os cuidadores e outros provedores necessidade de medicação pré-anestésica, evitar sintomas desagradáveis no pósoperatório, como náuseas e vômitos, usando tratamento farmacológico adequado. Por fim, manter o contato próximo dos pacientes com os cuidadores nos momentos de retorno da consciência, evitando assim agitação e ansiedade. Para aquela autora, descobertas recentes à publicação reforçavam a necessidade de um estudo rigoroso dos problemas potenciais que as crianças autistas poderiam ter quando submetidas a procedimentos anestésicos ${ }^{4}$.

Taghizadeh e colaboradores comentaram que os TEA seriam diagnosticados em pelo menos uma a cada 100 crianças até o momento da publicação do trabalho (2015), portanto, não seria surpreendente que os anestesistas estivessem cada vez mais atentos às crianças com esse diagnóstico. A classificação diagnóstica para TEA teria mudado e a compreensão médica das causas e gestão de TEA também teriam se adaptado a tais mudanças. Tendo em vista tal cenário, esta revisão tinha como objetivo fornecer uma atualização oportuna para aumentar a compreensão e consciência dos 
problemas que as crianças com TEA experimentariam, o que minimizaria os problemas perioperatórios que poderiam ocorrer por conta da citada condição. Os pesquisadores revisaram a literatura disponível sobre pré-medicação e o uso de alfa-2 agonistas, como clonidina e dexmedetomidina, bem como o uso de antigos favoritos midazolam e cetamina,sendo os trabalhos favoráveis ao uso dessas medicações, trazendo melhor experiência e aceitação para os pais e pacientes. 0 cuidado no pós-operatório também foi discutido, pois esses pacientes normalmente apresentam agitação intensa, após recobrarem a consciência. Foram descritas algumas estratégias simples que melhorariam o cuidado e diminuiriam a ansiedade dos pacientes, como histórias sociais, uso de tablets, jogos para distração, o uso de bebidas apreciadas pelas crianças para disfarçar o gosto amargo dos medicamentos e a necessidade de fazer o pós-operatório imediato em um local calmo, confortável e na presença dos cuidadores. $\mathrm{O}$ artigo frisou o fato de que os pais seriam os mais aptos a fornecerem informações sobre o que agitaria ou tranquilizaria seus filhos, sendo, portanto, ideal falar com eles pelo menos um dia antes doprocedimento. Os autores finalizam o trabalho reforçando a importância do treinamento das equipes e de diretrizes de prática clínica disponíveis em todas as instituições nos casos que envolvem crianças com TEA ${ }^{2}$.

Padish-Clarin e associados analisaram os resultados de processos que visavam reduzir a necessidade de uso de anestesia na avaliação da perda auditiva pediátrica por meio de uma revisão retrospectiva de prontuários que ponderavam a frequência com que uma avaliação auditiva pediátrica foi bem ou mal sucedida e um teste de resposta auditiva do tronco encefálico (RATE). Trinta porcento dos pacientes encaminhados para RATE concluíram com sucesso sua avaliação auditiva pediátrica, evitando, portanto, o procedimento anestésico, a maioria sendo do sexo feminino. Indivíduos do sexo masculino e indivíduos com atraso no desenvolvimento foram mais propensos a realizar o teste com anestesia. Paciente com TEA e atraso na fala tiveram baixas taxas de sucesso para realizar os testes sem anestesia, mostrando que essa é uma ferramenta útil para pacientes com TEA, até mesmo em procedimentos que habitualmente não necessitariam. Para esse procedimento foi utilizado sevoflurano ou sevoflurano com propofol intravenoso. Para os autores, os processos que foram implementados neste estudo resultaram na eliminação da necessidade do uso de anestesia para avaliação da audição em grande parte dos pacientes encaminhados. As taxas de sucesso para evitar a anestesia poderiam variar dependendo dos recursos disponíveis, educação do médico e população testada. Os benefícios adicionais do processo descrito incluiriam redução do estresse no sistema de saúde e nas famílias que receberiam os serviços, pois haveria alta mais precoce dos pacientes, retorno mais rápido ao trabalho pelos pais. Porém isso não acontece com os pacientes autistas, pois houve maior necessidade de uso de anestésicos por esses pacientes ${ }^{5}$.

Vlassakova e Emmanouil citaram que TEA era o distúrbio de neurodesenvolvimento que mais crescia no mundo e que o aumento do 
reconhecimento e da consciência pública sobre a doença teria sido impulsionado em grande parte pelos avanços na pesquisa médica. A literatura sobre anestesia consistiria principalmente de relatos de casos, porém, um número crescente de publicações estaria relatando estratégias de gerenciamento e avaliação das experiências perioperatórias dessa população de pacientes. Pacientes com TEA apresentam desafio na comunicação e na mudança de rotina, principalmente em ambientes não familiares. Aqueles com maior nível de gravidade, apresentam comportamentos que dificultam a interação com o anestesiologista. Então a conexão com a família e contanto prévio com opaciente são importantes para formação de vínculo e sucesso do procedimento. $O$ uso de medicação pré-anestésica (midazolam, cetamina ou dexmedetomidina) são opções favoráveis para que haja maior cooperação durante a indução anestésica, principalmente nos pacientes com comportamentos mais agressivos. Em resumo, pacientes com TEA seriam um grupo heterogêneo e frequentemente precisariam de anestesia para diferentes procedimentos e estudos. Sendo assim, os autores finalizaram salientando que a familiaridade com as especificidades comportamentais de cada paciente e esforços para aliviar seu estresse seriam de suma importância para um curso perioperatório tranquilo e com o mínimo de eventos adversos ${ }^{6}$.

Berglund e colaboradores desenvolveram diretrizes que melhorariam os cuidados de crianças com TEA, particularmente no que diz respeito à preparação dessas crianças para anestesia e procedimentos radiológicos. Para tanto, fizeram distribuição online de questionários, cujos resultados foram usados na elaboração de diretrizes para o cuidado de crianças com TEA. Vinte e um participantes, que trabalhavam com portadores de TEA em vários departamentos de anestesia e radiologia na Suécia, foram incluídos no painel de especialistas e uma lista de itens para o questionário foi criada a partir de uma pesquisa anterior e de literatura. Na primeira rodada, os itens com concordância $<60 \%$ foram descartados. Os itens restantes foram mesclados e uma nova lista foi criada. Mais duas rodadas semelhantes foram realizadas. Nas últimas 2 rodadas, 21 participantes responderam, e $80 \%$ de concordância foi considerada como consenso. Os autores expuseram que as diretrizes finais consistiram em catorze itens e uma lista de verificação de dezesseis fatores. As cinco áreas contempladas pelos itens e pelo checklist foram as seguintes: planejamento envolvendo pais / responsáveis, características do ambiente, otimização do tempo, comunicação e profissionais de saúde. A organização foi importante para possibilitar aos profissionais cuidar de cada criança de acordo com as necessidades específicas de cada caso e o envolvimento dos pais / responsáveis foi importante para obter conhecimentos sobre o comportamento da criança. Como conclusões, os pesquisadores relataram que as aplicações de anestesia e radiologia que envolvem crianças com TEA requereriam um planejamento prévio, atendendo especificamente às necessidades individuais de cada criança. Para isso, seria necessário que os profissionais de saúde tivessem conhecimentos básicos sobre os TEA e sua manifestação particular na criança confiada aos seus cuidados e a organização precisaria ter estruturas estabelecidas para facilitar esse processo ${ }^{7}$. 
Swartz e associados determinaram a utilidade de um plano individualizado com base na decisão de fornecer sedação pré-operatória estratificada pelo nível de gravidade dos TEA e a eficácia deste plano com base na avaliação subjetiva do comportamento do paciente durante a indução da anestesia e na satisfação do cuidador. Para tanto, revisaram a importância de um plano individualizado para cada criança com TEA que foram encaminhadas para anestesia. As crianças foram categorizadas pelo nível de gravidade dos TEA e, com a aprovação comitê de ética, os autores conduziram uma revisão retrospectiva dos prontuários para documentar a necessidade de sedação préoperatória, sedação estratificada pelo nível de gravidade do transtorno apresentado pelo paciente, comportamento na indução e satisfação do cuidador. Os autores relatam que entre 2012 e 2014 elaboraram com sucesso um plano para 246 de 251 procedimentos cirúrgicos ou diagnósticos em 224 pacientes. O nível de severidade foi de $45 \%$ de severidade Nível 1 e Asperger, 25\% de severidade Nível 2 e 30\% de severidade Nível 3. A maioria doscasos foi agendada para cirurgia diurna. A sedação pré-operatória foi administrada por via oral (VO), intramuscular (IM) ou intravenoso (IV). O esquema VO foi feito em 30 pacientes com $0,1 \mathrm{mg} / \mathrm{kg}$ de diazepam, 0,5mg/kg de midazolam ou $1,5 \mathrm{mg} / \mathrm{kg}$ de cetamina associada a $0,25 \mathrm{mg} / \mathrm{kg}$ de midazolam. Já o esquema IM foi realizado em 42 pacientes com $3,5 \pm 1.1 \mathrm{mg} / \mathrm{kg}$ de cetamina. E o esquema IV foi realizado em 21 paciente, titulando propofol com midazolam. A utilização de sedação aumentou com o nível de gravidade: nível de gravidade 1 (21\%) ou Asperger (31\%), nível de gravidade 2 (44\%) e nível de gravidade 3 (56\%). O odds ratio para o uso de sedação foi de 5,5 [IC: 2,6-11,5, P <0,001] com pacientes de nível de gravidade 3 versus nível de gravidade 1 . A cooperação na indução da anestesia foi de $90 \%$ no geral com ou sem sedação. Sendo esta administrada a 94 crianças (38\%). A cooperação com o procedimento foi maior no nível de gravidade 1 e em pacientes com Asperger, e um pouco menos em pacientes nos níveis de gravidade 2 e 3 . Pa ra os autores, o plano foi útil para orientar as escolhas de sedação, visto que a cooperação não diferiu entre sedados e crianças não sedadas em qualquer nível de gravidade. A satisfação entre os cuidadores foi de $98 \%$. Tais resultados sugeririam que um plano individualizado seria útil no manejo perioperatório de crianças com TEA e que o conhecimento do nível de gravidade do transtorno poderia ser útil na determinação da necessidade de sedação pré-operatória ${ }^{8}$.

Kamat e colaboradores visaram determinar como a sedação em procedimentos médicos seria fornecida a crianças com TEA e se os programas de sedação teriam protocolos especializados para essas crianças. Para tanto, os autores entraram em contato com médicos diretores de programas de sedação que são membros da Society for Pediatric Sedation e perguntaram sobre as características da prática e a utilização de recursos durante o procedimento de sedação de crianças com TEA. Dos 58 diretores, 47 responderam e dos programas pesquisados, 53\% eram grandes centros médicos universitários e 40\% eram hospitais infantis independentes. Apenas 25,5\% dos programas usaram um plano individualizado para autismo.Para realizar a sedação 
nesta coorte de estudo, 36\% dos programas usaram enfermeiras adicionais, enquanto um especialista em vida infantil foi usado em $55 \%$ dos programas pesquisados. Apenas $28 \%$ dos centros alocaram tempo adicional para acomodar crianças com TEA. Os métodos de distração foram usados em $80 \%$ dos casos, enquanto que a restrição ocorreu em $45 \%$ dos programas, geralmente quando se necessitava de colocação do cateter intravenoso, principalmente em caso de pacientes com maior gravidade de TEA. Também se observou maior necessidade de uso de medicação pré-anestésica, nesse grupo de pacientes, como midazolam oral ou intranasal, cetamina intramuscular ou dexmedetomidina intranasal. Para a sedação durante o procedimento, propofol foi o agente preferido por $70 \%$ dos programas de imagem, enquanto o propofol associado ao fentanil foi usado por $66 \%$ dos programas para procedimentos dolorosos. Em relação a efeitos adversos pós-anestesia, os participantes da pesquisa não notavam diferença em relação a pacientes não-autistas, tanto para agitação como para delirium. Embora 57\% dos diretores tenham relatado que a equipe do programa ficava extremamente confortável fornecendo sedação para crianças com TEA, 79\% dos diretores desejavam mais educação sobre estratégias de gerenciamento comportamental para sedação dessas crianças. Os pesquisadores concluíram que entre os programas da Society for Pediatric Sedation existia uma variação significativa na aplicação de sedação para crianças com TEA. Uma melhor compreensão dos recursos necessários, a padronização de estratégias de gestão comportamental e abordagens farmacológicas e o desenvolvimento de protocolos poderiam ajudar a otimizar o atendimento a essa população, evitando experiências traumáticas e inseguras ${ }^{9}$.

Para Abulebda e colaboradores crianças com TEA teriam uma alta incidência de comorbidades neurológicas. Consequentemente, a avaliação com ressonância magnética (RM) seria considerada necessária, porém, sedar esses pacientes apresentaria vários desafios. Portanto, este estudo retrospectivo comparou a eficácia e segurança da dexmedetomidina ao propofol em pacientes autistas que foram sedados e submetidos à RM. Foram avaliados 56 pacientes no grupo dexmedetomidina e 49 no grupo propofol e todos concluíram o procedimento com sucesso. O grupo da dexmedetomidina recebeu dose de indução de $2 \mathrm{mcg} / \mathrm{kg}$ em $10 \mathrm{~min}$, com dose de manutenção de $1 \mathrm{mcg} / \mathrm{kg} / \mathrm{h}$. Dose adicional de $2 \mathrm{mcg} / \mathrm{kg}$ foi feita em pacientes que não ficaram bem sedados após a primeira dose de indução. $O$ grupo de propofol, a dose de indução foi de $1 \mathrm{mg} / \mathrm{kg}$ (máximo de $50 \mathrm{mg}$ ), com dose de manutenção de $83 \mathrm{mcg} / \mathrm{kg} / \mathrm{min}$. Se ocorreu movimentação indesejada durante o procedimento doses adicionais de 0.5 $1 \mathrm{mg} / \mathrm{kg}$ dos respectivos medicamentos eram utilizadas. Os tempos indução, recuperação e alta foram significativamente menores no grupo propofol, enquanto o grupo dexmedetomidina manteve a hemodinâmica mais estável. Tanto o propofol quanto à dexmedetomidina mostraram-se medicamentos adequados e seguros na sedação de crianças autistas submetidas à $\mathrm{RM}^{10}$. 
Ohtawa e colaboradores pesquisaram a satisfação dos pais com a anestesia ambulatorial durante o tratamento odontológico em pacientes com comportamento neurológico atípico. Fatores associados à preferência dos pais por anestesia geral durante o tratamento odontológico futuro em tais pacientes também foram investigados. Um questionário foi enviado aos pais de 181 portadores de transtornos mentais submetidos a tratamento odontológico sob anestesia ambulatorial no Tokyo Dental College of Suidobashi Hospital entre 2012 e 2016 e um total de 71 respostas foram recebidas (39,2\%). A idade média dos pacientes foi de 18 anos e, as deficiências incluíam TEA, deficiência intelectual, paralisia cerebral e epilepsia. Os itens pesquisados incluíram detalhes do tratamento odontológico, número de vezes que os pacientes receberam anestesia geral, tipo de anestésico utilizado, método de indução da anestesia, duração do tratamento e da anestesia e a presença ou ausência de complicações intra ou pós-operatórias. Pesquisou-se, ainda, problemas relacionados ao atendimento odontológico, histórico de anestesia, ansiedade pré-operatória, tempo de jejum, indução de anestesia geral, enfermagem e ambiente de quarto hospitalar, ansiedade pós-operatória, avaliação geral e se os pais prefeririam anestesia geral durante o tratamento odontológico futuro. Os pacientes foram divididos em 2 grupos: aqueles cujos pais preferiram a anestesia geral durante o futuro tratamento odontológico e aqueles cujos pais não preferiram. O resultado mostrou que a maioria dos pais dos indivíduos com deficiência que receberam anestesia geral anteriormente durante o tratamento odontológico preferiram a anestesia geral durante o tratamento odontológico futuro. Os que não preferiram a anestesia nos tratamentos futuros, não deixaram claras suas objeções em relação à preferência, ousimplesmente não queriam que os filhos se submetessem à anestesia geral. Quase metade dos pais relataram que o início da anestesia não foi de forma tranquila, tanto por comportamento do paciente como por inadequação do material utilizado (ex. máscara facial inadequada para o paciente). O momento da indução pode gerar uma impressão negativa para os cuidadores, por isso é importante explicar como será o procedimento, bem como pode ser a reação do paciente nesse momento, gerando assim menos angústia e ansiedade para os pais e pacientes. Além disso há algumas razões que possam explicar a preferência de alguns pais em não realizar a anestesia, como: complicações ou acidentes relacionados a anestesia, o impacto das drogas no organismo dos indivíduos e a necessidade de jejum pré-procedimento, devendo então ser explicados todo o procedimento, tanto odontológico como anestésico, explicitando os riscos e benefícios. O trabalho concluiu que a maioria dos pais preferiram que seus filhos fossem anestesiados novamente em um futuro tratamento odontológico ${ }^{11}$.

Mellado-Cairet e associados iniciaram seu trabalho comentando que crianças com TEA teriam maior probabilidade de ficarem angustiadas durante a indução da anestesia e que a indução inalatória seria quase sempre a via preferida, com a aceitação da máscara facial frequentemente se apresentando como um desafio considerável. Além disso, medidas de moderação para facilitar a anestesia por agentes inalatórios, 
como pré-medicação forçada e restrição física, não seriam mais opções viáveis, exceto em circunstâncias atenuantes. $O$ interesse de pesquisas recentes a esta publicação se concentrava na necessidade de planejamento avançado em colaboração com o responsável pela criança para adaptar um plano perioperatório individualizadocom intervenções farmacológicas e não farmacológicas. As estratégias de análise de comportamento aplicadas teriam uma eficácia bem documentada nesta população mudando sistematicamente o comportamento usual destes indivíduos. Deste modo, os autores apresentaram um relato de caso bem-sucedido de dessensibilização préoperatória de uma criança com TEA grave, onde foi usada uma técnica de demonstração em espelho associada ao reforço positivo para prepará-la para a anestesia geral. Também foram discutidas as aplicações potenciais de estratégias de análise de comportamento para anestesia nesta população. Os autores concluíram que de um ponto de vista prático, a comunicação precoce com os cuidadores seria necessária para estabelecer que pacientes poderiam se beneficiar desse treinamento comportamental e que em caso positivo, a preparação individual planejada para a anestesia geral deveria ser fornecida por uma equipe multidisciplinar treinada ${ }^{12}$.

Whippey e colaboradores realizaram um estudo piloto de viabilidade de cuidados perioperatórios para pacientes com TEA a serem anestesiados. Para tanto, utilizaram feedbacks dos pais e dos provedores e elaboraram um protocolo que incluía modificações do ambiente, planos de ansiólise, conjuntos de pedidos especializados e suporte de especialistas na vida infantil (SEVI) que foram desenvolvidos ao longo de um período de nove meses. Scores de gravidade do autismo (SGA), estilos de comunicação, gatilhos e experiências anteriores foram usados para criar planos de cuidados individualizados na clínica pré-operatória. Scores de emoção e sedação na unidade de cirurgia no dia do procedimento, na indução da anestesia e na sala de recuperação pósanestésica foram registrados. A aceitação do protocolo foi obtida por enfermeiros, anestesiologistas e pais. Os critérios de viabilidade incluíram a taxa de recrutamento, adesão ao protocolo, coleta de dados e acompanhamento do paciente. Como resultado, os autores relataram que dezoito pacientes foram incluídos neste estudo piloto e que todos os critérios de viabilidade, incluindo recrutamento, adesão ao protocolo do estudo (97\%) e acompanhamento (94\%) foram atendidos. Quinze pacientes eram não verbais e minimamente interativos (SGA $=3$ ). Após a implementação do protocolo, 15 das induções anestésicas foram descritas como excelentes. Dez planos de prémedicação, incluindo diferentes combinações de midazolam e cetamina foram usados. O plano de medicação variava de acordo com a individualidade de cada paciente, como a sensibilidade ao gosto, experiência prévia com alguma medicação e o nível esperado de cooperação do paciente. Os pais descreveram o plano personalizado, a medicação ansiolítica e o SEVI como vantajosos. Adicionalmente, sugeriram incorporar ao protocolo o uso de ferramentas que melhorassem a comunicação visual com esses pacientes, mais informações específicas aos pais de pacientes com TEA e reforçaram a necessidade de consistência nas visitas. Todos enfermeiros, anestesiologistas e pais 
achavam que o programa deveria continuar. Com isso, os pesquisadores concluíram que um plano de cuidados perioperatório multidisciplinar para crianças com TEA grave seria viável e altamente aceito na instituição onde o estudo ocorreu. A natureza individual dos planos de ansiólise foi considerada um ponto forte do protocolo ${ }^{13}$.

\section{CONCLUSÃO}

Pacientes com TEA são frequentemente submetidos a procedimentos sob anestesia, necessitando, pois, de atenção especial da equipe. A falta de contato prévio com paciente e familiares e procedimentos pré-operatórios, como a contenção física para facilitar aplicações anestésicas, já não são mais eticamente bem vistos, e é crescente a necessidade de protocolos que abrangem os efeitos da TEA nestes pacientes para que estratégias de contenção física seja utilizada apenas em último caso.

A interação maior com os pais ou cuidadores sobre as especificidades mentais de cada paciente geram bons resultados e o treinamento de profissionais da saúde sobre TEA e seus desdobramentos comportamentais são de extrema necessidade para que as anestesias, procedimentos cirúrgicos e exames obtenham o sucesso desejado. Estratégias simples que melhorariam o cuidado e diminuiriam a ansiedade dos pacientes, como histórias sociais, uso de tablets, jogos para distração, uso de bebidas apreciadas pelos pacientes para disfarçar o gosto amargo dos medicamentos e demonstração em espelho são associadas ao reforço positivo na preparação de crianças portadoras de TEA para a anestesia geral. Além disso, o uso de SGA foi considerado uma boa ferramenta para o planejamento do procedimento de anestesia de crianças com TEA pela equipe médica. O principal ponto levantado nos trabalhos foi a necessidade do conhecimento prévio dos pacientes com TEA, entendendo suas particularidades e formando um plano individualizado para cada paciente, muitas vezes prescindindo até de medicação pré-anestésica, quando se há uma boa interação médico-famíliapaciente.

Os anestésicos comumente citados pelos artigos incluem midazolam, cetamina, propofol, clonidina e dexmedetomidina, e se mostraram capazes de melhorar a dinâmica no processo anestesia-cirurgia, sem gerar tantos traumas para paciente e familiares. Por último, é importante notar que quanto menor a severidade dos TEA apresentados pelo paciente, maior é a chance de sucesso das estratégias aplicadas.

\section{REFERÊNCIAS}

1. Lord C, Brugha TS, Charman T, Cusack J, Dumas G, Frazier T, et al. Autism spectrum disorder. Nat Rev Dis Prim [Internet]. 2020 Jan 1 [cited 2021 Sep 21];6(1). Available from: https://pubmed.ncbi.nlm.nih.gov/31949163/

2. Taghizadeh N, Davidson A, Williams K, Story D. Autism spectrum disorder (ASD) and its perioperative management [Internet]. Vol. 25, Paediatric Anaesthesia. Paediatr 
Anaesth; 2015 [cited 2021 Sep 21]. p. 1076-84. Available from: https://pubmed.ncbi.nlm.nih.gov/26248302/

3. Capp PL, de Faria MEJ, Siqueira SRDT, Cillo MTP, Prado EGB, de Siqueira JTT. Special care dentistry: Midazolam conscious sedation for patients with neurological diseases. Eur J Paediatr Dent [Internet]. 2010 Dec;11(1):162-4. Available from: http://europepmc.org/abstract/MED/21250764

4. Bagshaw M. Anaesthesia and the autistic child. J Perioper Pract [Internet]. 2011 [cited 2021 Sep 21];21(9):313-7. Available from: https://pubmed.ncbi.nlm.nih.gov/22474776/

5. Padish-Clarin G, Hawkins AJ. Retrospective analysis of decreasing the use of anesthesia in pediatric audiology: A preliminary study. Am J Audiol [Internet]. 2015 Dec 1 [cited 2021 Sep 21];24(4):557-62. Available from: https://pubmed.ncbi.nlm.nih.gov/26650987/

6. Vlassakova BG, Emmanouil DE. Perioperative considerations in children with autism spectrum disorder [Internet]. Vol. 29, Current Opinion in Anaesthesiology. Curr Opin Anaesthesiol; 2016 [cited 2021 Sep 21]. p. 359-66. Available from: https://pubmed.ncbi.nlm.nih.gov/26914785/

7. Berglund IG, Björkman B, Enskär K, Faresjö M, Huus K. Management of Children with Autism Spectrum Disorder in the Anesthesia and Radiographic Context. J Dev Behav Pediatr [Internet]. 2017 Apr 1 [cited 2021 Sep 21];38(3):187-96. Available from: https://pubmed.ncbi.nIm.nih.gov/28333847/

8. Swartz JS, Amos KE, Brindas M, Girling LG, Ruth Graham M. Benefits of an individualized perioperative plan for children with autism spectrum disorder. Paediatr Anaesth [Internet]. 2017 Aug 1 [cited 2021 Sep 21];27(8):856-62. Available from: https://pubmed.ncbi.nlm.nih.gov/28618130/

9. Kamat PP, Bryan LN, McCracken CE, Simon HK, Berkenbosch JW, Grunwell JR. Procedural sedation in children with autism spectrum disorders: A survey of current practice patterns of the society for pediatric sedation members. Paediatr Anaesth [Internet]. 2018 Jun 1 [cited 2021 Sep 21];28(6):552-7. Available from: https://pubmed.ncbi.nlm.nih.gov/29732645/

10. Abulebda K, Louer R, Lutfi R, Ahmed SS. A Comparison of Safety and Efficacy of Dexmedetomidine and Propofol in Children with Autism and Autism Spectrum Disorders Undergoing Magnetic Resonance Imaging. J Autism Dev Disord [Internet]. 2018 Sep 1 [cited 2021 Sep 21];48(9):3127-32. Available from: https://pubmed.ncbi.nlm.nih.gov/29680960/

11. Ohtawa Y, Yoshida M, Fukuda K. Parental Satisfaction with Ambulatory Anesthesia during Dental Treatment for Disabled Individuals and Their Preference for Same in Future. Bull Tokyo Dent Coll [Internet]. 2019 Feb 28 [cited 2021 Sep 21];60(1):5360. Available from: https://pubmed.ncbi.nIm.nih.gov/30700644/

12. Mellado-Cairet $P$, Harte $C$, Séjourné $E$, Robel L. Behavioral training and mirroring techniques to prepare elective anesthesia in severe autistic spectrum disorder patients: An illustrative case and review. Paediatr Anaesth. 2019;29(3):226-30. 
Considerações anestésicas em pacientes pediátricos com transtorno do espectro autista

Dibe AGM, et al

13. Whippey A, Bernstein LM, O'Rourke D, Reddy D. Enhanced perioperative management of children with autism: a pilot study. Can J Anesth. 2019;66(10):1184-93. 